\title{
Investigation of p-type depletion doping for InGaN/GaN-based light-emitting diodes
}

Yiping Zhang, Zi-Hui Zhang, Swee Tiam Tan, Pedro Ludwig Hernandez-Martinez, Binbin Zhu, Shunpeng Lu, Xue Jun Kang, Xiao Wei Sun, and Hilmi Volkan Demir

Citation: Appl. Phys. Lett. 110, 033506 (2017); doi: 10.1063/1.4973743

View online: https://doi.org/10.1063/1.4973743

View Table of Contents: http://aip.scitation.org/toc/apl/110/3

Published by the American Institute of Physics

\section{Articles you may be interested in}

GaN-based light emitting diodes using p-type trench structure for improving internal quantum efficiency Applied Physics Letters 110, 021115 (2017); 10.1063/1.4973995

On the mechanism of highly efficient p-type conduction of Mg-doped ultra-wide-bandgap AIN nanostructures Applied Physics Letters 110, 032102 (2017); 10.1063/1.4973999

The effect of polarity on MOCVD growth of thick InGaN

Applied Physics Letters 110, 022101 (2017); 10.1063/1.4972967

All metalorganic chemical vapor phase epitaxy of $p / n-G a N$ tunnel junction for blue light emitting diode applications

Applied Physics Letters 110, 102104 (2017); 10.1063/1.4978268

Determination of the radiative efficiency of GaN-based light-emitting diodes via bias dependent resonant photoluminescence

Applied Physics Letters 110, 011106 (2017); 10.1063/1.4973589

MBE-grown 232-270 nm deep-UV LEDs using monolayer thin binary GaN/AIN quantum heterostructures Applied Physics Letters 110, 041108 (2017); 10.1063/1.4975068

\section{PHYSICS TODAY}

WHITEPAPERS
MANAGER'S GUIDE

Accelerate R\&D with

Multiphysics Simulation
READ NOW

PRESENTED BY

И๐OMSOL 


\title{
Investigation of p-type depletion doping for InGaN/GaN-based light-emitting diodes
}

\author{
Yiping Zhang, ${ }^{1, a)}$ Zi-Hui Zhang, ${ }^{1,2, a)}$ Swee Tiam Tan, ${ }^{1}$ Pedro Ludwig Hernandez-Martinez, ${ }^{1}$ \\ Binbin Zhu, ${ }^{1}$ Shunpeng Lu, ${ }^{1}$ Xue Jun Kang, ${ }^{1}$ Xiao Wei Sun, ${ }^{3, b)}$ \\ and Hilmi Volkan Demir ${ }^{1,4,5, b)}$ \\ ${ }^{1}$ Luminous! Centre of Excellence for Semiconductor Lighting and Displays, School of Electrical and \\ Electronic Engineering, Nanyang Technological University, 50 Nanyang Avenue, Singapore 639798, \\ Singapore \\ ${ }^{2}$ Key Laboratory of Electronic Materials and Devices of Tianjin, School of Electronics and Information \\ Engineering, Hebei University of Technology, 5340 Xiping Road, Beichen District, Tianjin 300401, \\ People's Republic of China \\ ${ }^{3}$ Department of Electrical and Electronic Engineering, College of Engineering, Southern University of Science \\ and Technology, No 1088, Хueyuan Rd, Nanshan District, Shenzhen, Guangdong 518055 , \\ People's Republic of China \\ ${ }^{4}$ School of Physics and Mathematical Sciences, Nanyang Technological University, 21 Nanyang Link, \\ Singapore 637371, Singapore \\ ${ }^{5}$ Department of Electrical and Electronics, Department of Physics, and UNAM-National Nanotechnology \\ Research Center and Institute of Material Science and Nanotechnology, Bilkent University, Ankara TR-06800, \\ Turkey
}

(Received 26 September 2016; accepted 23 December 2016; published online 20 January 2017)

\begin{abstract}
Due to the limitation of the hole injection, p-type doping is essential to improve the performance of InGaN/GaN multiple quantum well light-emitting diodes (LEDs). In this work, we propose and show a depletion-region Mg-doping method. Here we systematically analyze the effectiveness of different $\mathrm{Mg}$-doping profiles ranging from the electron blocking layer to the active region. Numerical computations show that the Mg-doping decreases the valence band barrier for holes and thus enhances the hole transportation. The proposed depletion-region Mg-doping approach also increases the barrier height for electrons, which leads to a reduced electron overflow, while increasing the hole concentration in the p-GaN layer. Experimentally measured external quantum efficiency indicates that $\mathrm{Mg}$-doping position is vitally important. The doping in or adjacent to the quantum well degrades the LED performance due to $\mathrm{Mg}$ diffusion, increasing the corresponding nonradiative recombination, which is well supported by the measured carrier lifetimes. The experimental results are well numerically reproduced by modifying the nonradiative recombination lifetimes, which further validate the effectiveness of our approach. Published by AIP Publishing.
\end{abstract}

[http://dx.doi.org/10.1063/1.4973743]

Despite drastic progress in research and commercialization being made in the past decades, GaN-based light-emitting diodes (LEDs) are still suffering from a serious efficiency droop, especially at high current density injection. Hole transportation is claimed to be one of the most important factors for causing the efficiency droop, such as nonuniform hole distribution in the multiple quantum wells (MQWs) and the low hole injection efficiency. ${ }^{1-3}$ Consequently, tremendous efforts have been devoted to address these issues to improve the LED performance. As a result, structures such as engineered AlGaN electron-blocking layer (EBL), ${ }^{3-5}$ p-type InGaN hole reservoir layer, ${ }^{6}$ hole modulator by p-type doped last quantum barrier (QB), ${ }^{7}$ AlGaN polarization doping, ${ }^{8-10}$ and $\mathrm{p}$-doped $\mathrm{QBs},{ }^{11,12}$ etc., have been reported to enhance hole injection and reduce the efficiency droop. However, there is a lack of systematic investigations on how the p-type doping at different regions for the LED device influences the carrier transportation and hence the carrier recombination.

It is well known that the EBL is commonly adopted for InGaN/GaN LED structures to suppress the electron

\footnotetext{
${ }^{\text {a) }}$ Y. Zhang and Z.-H. Zhang contributed equally to this work.

b) Electronic addresses: exwsun@ntu.edu.sg and volkan@stanfordalumni.org
}

overflow. However, an undoped AlGaN EBL significantly impedes the hole transport into active region. Thus the EBL has to be p-type which can then efficiently reduce electron leakage while decreasing the potential barrier height for the holes. ${ }^{13}$ Kuo et al. report that the InGaN/GaN MQW LEDs with a partially p-doped last barrier exhibit the enhanced optical performance, and they attribute this improvement to the increased hole injection and the larger effective barrier height for electrons. ${ }^{14}$ Han et al. suggest that an enhanced performance can be obtained even at the high current for InGaN/GaN LEDs with Mg-doped quantum barriers, and the better performance is due to the improved hole injection caused by the modification of the energy bands. Moreover, they suggest that the Mg-doped QBs can improve the morphological properties. ${ }^{15}$ On the other hand, Köhler et al. report the damaged active region due to the $\mathrm{Mg}$ diffusion and migration, especially when $\mathrm{Mg}$-doped position is designed near to the active region. These in turn increases the nonradiative recombination in the quantum well (QW) active region and reduces the output power. ${ }^{16}$ Despite various reports on the effect of $\mathrm{Mg}$-doping on the LED performance, there is still no conclusive consensus that can be drawn. As a result, a systematic study is strongly needed to 
uncover the underlying physics. Hence, in this work, we propose the depletion-region doping to systematically analyze the $\mathrm{Mg}$-doping effect on an active region for blue-emitting InGaN/GaN MQW LEDs and investigate the underlying physics in conjunction with theoretical modeling. We find that $\mathrm{Mg}$-doping at different ranges for the depletion region affects the energy band barriers, carrier injection, and even the optical output power.

Five InGaN/GaN MQW LED wafers used in this work were grown on (0001) c-plane sapphire substrates using a metal-organic chemical-vapor deposition (MOCVD) system. The growth was initiated on a 30-nm thick low-temperature $\mathrm{GaN}$ nucleation layer followed by a $4-\mu \mathrm{m}$ unintentionally doped n-type GaN (u-GaN) layer. Then a Si-doped n-GaN layer was grown on the u-GaN template with the $\mathrm{Si}$ doping concentration and thickness of $5 \times 10^{18} \mathrm{~cm}^{-3}$ and $2-\mu \mathrm{m}$, respectively. Subsequently, 8 pairs of $\mathrm{In}_{0.15} \mathrm{GaN}_{0.85} / \mathrm{GaN}$ MQWs were grown with 3-nm thick quantum well and 9-nm thick quantum barrier. After the MQWs, all LED samples were capped with a $20-\mathrm{nm} \mathrm{Al}_{0.15} \mathrm{Ga}_{0.85} \mathrm{~N}$ EBL and a $150-\mathrm{nm}$ thick $\mathrm{p}-\mathrm{GaN}$ layer. The difference among these five LED samples comes from the p-type doped position, such that, p-type doping is at EBL solely, EBL $+1 / 2 \mathrm{QB}, \mathrm{EBL}+\mathrm{QB}$, $\mathrm{EBL}+\mathrm{QB}+\mathrm{QW}$ and $\mathrm{EBL}+\mathrm{QB}+\mathrm{QW}+\mathrm{QB}$, as shown in Fig. 1. For instance, for LED with p-type doping at $\mathrm{EBL}+\mathrm{QB}+\mathrm{QW}+\mathrm{QB}$, when the growth of 7th QW is finished, the Mg precursors are opened until the growth of the EBL layer is completed with a flow rate of cyclopentadienyl magnesium $\left(\mathrm{Cp}_{2} \mathrm{Mg}\right)$ of $0.239 \mu \mathrm{mol} / \mathrm{min}$, and the estimated effective hole concentration is $5 \times 10^{17} \mathrm{~cm}^{-3}$. After EBL layer growth is completed, the $\mathrm{Cp}_{2} \mathrm{Mg}$ flow rate is set to $0.432 \mu \mathrm{mol} / \mathrm{min}$ with the estimated effective hole concentration of $1 \times 10^{18} \mathrm{~cm}^{-3}$ for the following $\mathrm{p}-\mathrm{GaN}$ layer growth. Finally, the in-situ $720^{\circ} \mathrm{C}$ thermal annealing treatment in $\mathrm{N}_{2}$ ambient was carried out to activate the Mg dopants. In order to precisely reveal the mechanism of how Mg-doping in the depletion region influences the LED performance, numerical simulations were conducted by Advanced Physical Models of Semiconductor Devices (APSYS) simulator and the parameter settings can be found in the supplementary material.

When a p-n junction is formed, the carriers will redistribute due to the large carrier concentration contrast until the equilibrium state is built up. There will be a depletion region in which no free carriers exist, and a built-in electric field is established. The width of the depletion region can be obtained by ${ }^{17}$
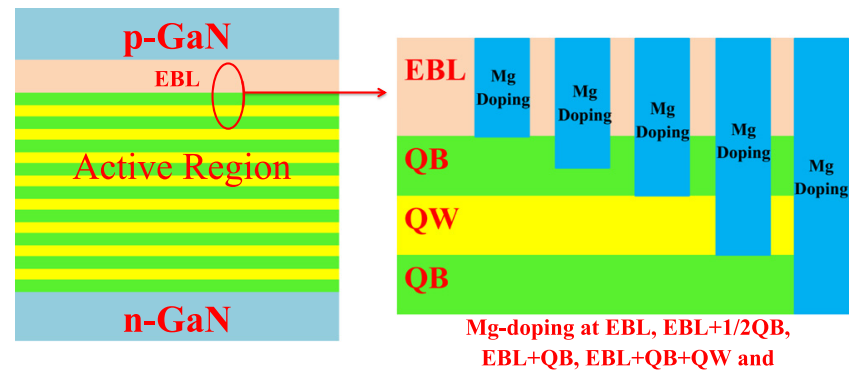

$\mathbf{E B L}+\mathbf{Q B}+\mathbf{Q W}+\mathbf{Q B}$

FIG. 1. Schematic diagram of different p-type doped configurations for InGaN/GaN LEDs.

$$
w_{d}=\sqrt{\frac{2 \varepsilon}{e} V_{D}\left(\frac{1}{N_{A}}+\frac{1}{N_{D}}\right)},
$$

where $\varepsilon$ is the dielectric permittivity, $V_{D}$ is the diffusion voltage, $N_{A}$ is the acceptor concentration, and $N_{D}$ is the donor concentration. It is worth mentioning that the actual depletion region contains two parts: (1) transition region and (2) completely depleted region. In order to probe the exact location of depletion region, we first performed numerical simulations to calculate the bandgap energy diagram and carrier concentration profiles for InGaN/GaN MQW LEDs when the device is under equilibrium state, as presented in Fig. 2.

The electron and hole concentration distribution in Fig. 2(b) clearly indicates that the depletion region completely depletes the electrons and holes. As displayed in Fig. 2(b), the electron concentration drops significantly and becomes negligible at depletion region along the growth orientation, while the hole concentration falls down rapidly and becomes negligible at depletion region along the [000-1] direction. The depletion region lies from half of the last QB to the half of EBL and its corresponding width is $15 \mathrm{~nm}$ in this case, which is illustrated in Fig. 2(a) by grey colour. Since the different p-type doping architectures such as p-type doped EBL and QB always affect the depletion region, investigating the link between the p-type doping and the depletion region will help to understand the working mechanisms for LEDs with various p-type doped configurations. Therefore, numerically
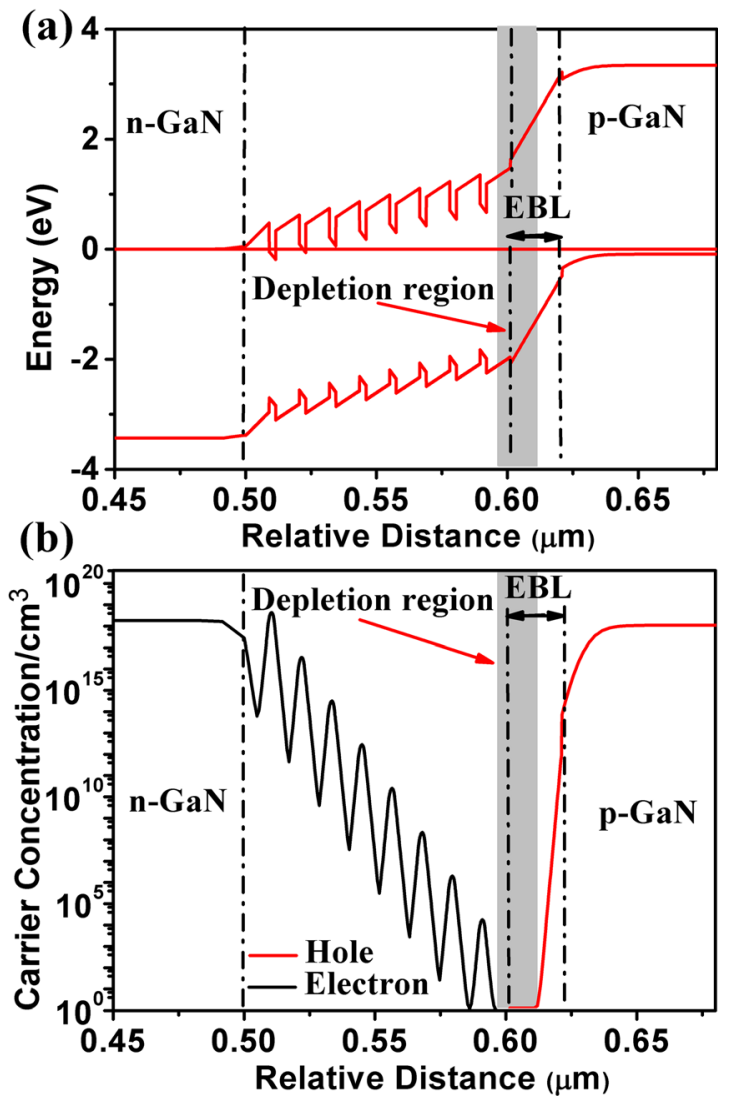

FIG. 2. (a) Schematic energy bandgap diagrams of depletion region for InGaN/GaN MQW LEDs under equilibrium state, (b) computed electron and hole concentration profiles in InGaN/GaN MQW LEDs. 
(a)

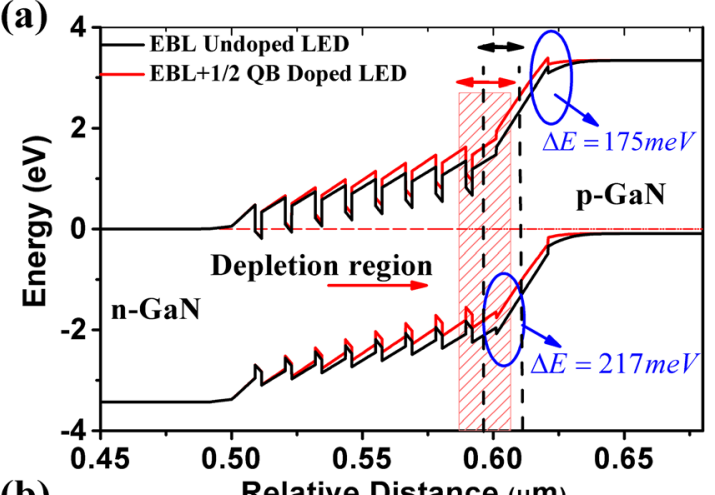

(b)

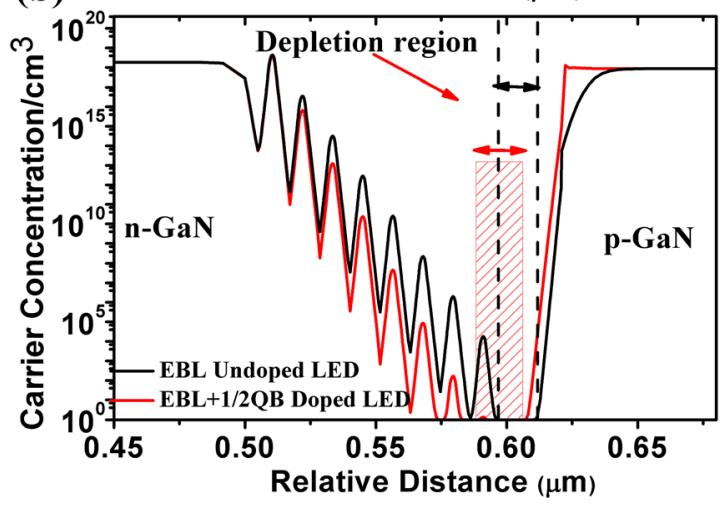

FIG. 3. Comparison of (a) bandgap diagram and (b) carrier distribution for EBL undoped LED and EBL $+1 / 2 \mathrm{QB}$-doped LED at equilibrium.

computed bandgap diagrams and carrier distributions are carried out for LED with Mg-doping at EBL $+1 / 2 \mathrm{QB}$.

Fig. 3 presents the comparison results of LEDs with and without depletion-region $\mathrm{Mg}$-doping layout, respectively. Here, it is worth noting that the EBL is totally doped. As indicated in Fig. 3(a), if the EBL $+1 / 2 \mathrm{QB}$ p-type doping is adopted, the barrier height for electron is increased by $175 \mathrm{meV}$ while the barrier height for hole is reduced by $217 \mathrm{meV}$ compared to EBL undoped LED, which is consistent with the previous reports of p-EBL LEDs. ${ }^{8,13}$ Moreover, according to the carrier distribution in Fig. 3(b), it is observed that the depletion region moves towards to the $\mathrm{n}-\mathrm{GaN}$ and the corresponding width gets larger with a value of $19 \mathrm{~nm}$. More importantly, the hole concentration in the $\mathrm{p}-\mathrm{GaN}$ at the interface between the $\mathrm{p}-\mathrm{GaN}$ layer and EBL is increased for EBL $+1 / 2 \mathrm{QB}$ p-doped LED. Because the holes in the p-doped $\mathrm{QB}$ are depleted by the net field which is the coupled effect of the built-in electric field and polarization induced electric field, and the holes are stored in the p-GaN layer, which correspondingly increases the overall hole concentration in the $\mathrm{p}$-GaN layer and enhances the hole injection into the active region. ${ }^{7}$

Due to the lattice mismatch between $\mathrm{InN}, \mathrm{GaN}$, and AlN, polarization is caused in the interfaces of QW and QB, and QB and EBL. Consequently, there are two electric fields existing in the depletion region. As indicated in Fig. 4(a), the orientation of the built-in electric field of depletion region is directed from n-side layer to $\mathrm{p}$-side layer and the orientation of strain-induced electric field in QB and EBL is in the same direction as built-in electric field. However, the polarization induced electric field in the QW region is opposite to the built-in electric field. If the whole depletion region is designed to be p-doped, the holes in this region will move due to the electric field. Fig. 4(a) presents the schematic diagram of possible movements of holes if the depletion region is designated to be p-type doping. For the holes in area (4) as shown in Fig. 4(a), they will move to area (5) driven by builtin electric field and strain-induced electric field. The holes then will finally migrate into the p-GaN layer, thus contributing to the enhanced hole concentration. For area (3), the holes will gain the energy from both the polarization induced electric field and the built-in field, and they will move from the original site to the interface of GaN last quantum barrier and AlGaN EBL. If the holes can obtain energy larger than the barrier for the GaN/AlGaN interface, they are able to climb over $\mathrm{p}$-EBL and finally reach the $\mathrm{p}-\mathrm{GaN}$ layer. On the other hand, the holes will be depleted into the nearest QW and recombine by nonradiative recombination if the obtained energy is not sufficient. As a result, the EBL $+1 / 2$ QB LED obtains a higher hole concentration in the $\mathrm{p}-\mathrm{GaN}$ which contributes to an enhanced overall hole injection efficiency. ${ }^{7}$ However, for the p-type doping in areas (1) and (2), holes cannot be depleted to $\mathrm{p}-\mathrm{GaN}$ layer due to the high barrier at QW/QB interface. Moreover, strain-induced electric field in QW does not favour the hole diffusion into the $\mathrm{p}-\mathrm{GaN}$ layer. Figure 4(b) displays the hole concentration profiles for LEDs with different Mg-doping positions, which support the explanation of hole migration into p-GaN.
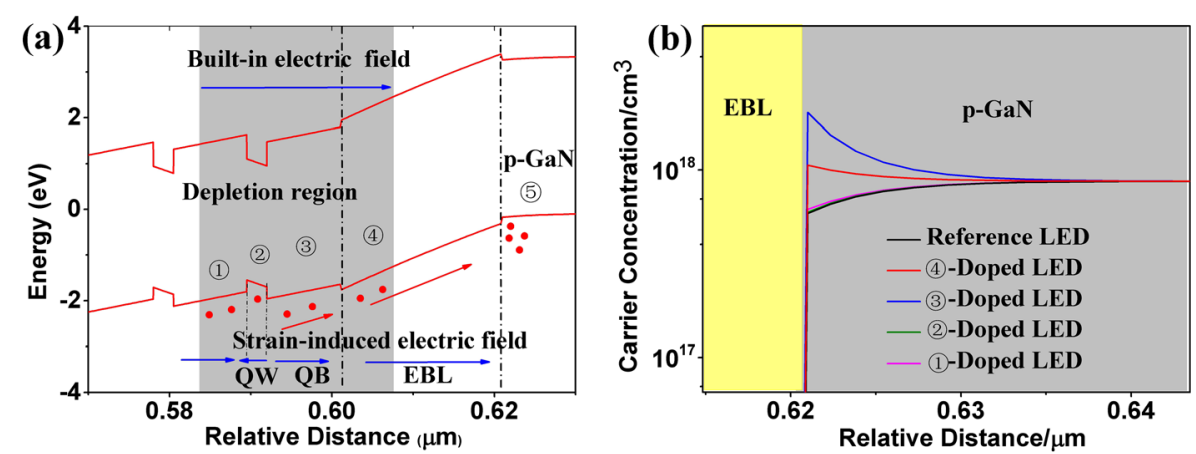

FIG. 4. (a) Schematic bandgap diagram around depletion region with built-in electric field and strain-induced electric field noted, (b) the hole concentration profiles at EBL/p-GaN interface for LEDs with different Mg-doping positions. The hole movement is indicated if the depletion region is p-doped. (1), (2), (3), (4), and (5) denote the area of QB, QW, QB, EBL, and p-GaN, respectively. Holes obtaining a sufficient energy in area (3) and (4) overcome the barrier and transport to p-EBL, and then transport to and store in (5) together with holes in p-EBL. Holes with a less energy in area (1) and (2) cannot be depleted to area (5), and will finally be eliminated by nonradiative recombination when the equilibrium state is built. 
To precisely probe the effectiveness of depletion-region Mg-doping approach, we have measured and demonstrated the optical output power for the five InGaN/GaN MQW LEDs with $\mathrm{Mg}$-doping at $\mathrm{EBL}, \mathrm{EBL}+1 / 2 \mathrm{QB}, \mathrm{EBL}+\mathrm{QB}$, $\mathrm{EBL}+\mathrm{QB}+\mathrm{QW}$, and $\mathrm{EBL}+\mathrm{QB}+\mathrm{QW}+\mathrm{QB}$, respectively. The experimental optical output power is measured by an Ocean Optics Spectrometer attached to the integrating sphere and is shown in Fig. 5(a). It is worth mentioning that the EBL and 2nd QB are full doped in order to cover the total area of the depletion region. As displayed in Fig. 5(a), the depletion-region Mg-doping approach strongly influences the LED performance. The trend of increasing the p-type doping range is not consistent with what we expect that a longer p-type doping region can lead to better performance. The LED with p-type doping at EBL $+1 / 2 \mathrm{QB}$ performs best, which is attributed to the reduced hole barrier and enhanced hole concentration in $\mathrm{p}-\mathrm{GaN} .^{7,14}$ Therefore, $\mathrm{p}$-type doping in this region is proved to enhance the hole transportation, hence improving the LED performance. When the p-type doping area in depletion region increases to the full $\mathrm{QB}$, the performance does not further increase as we would expect, which is attributed to the $\mathrm{Mg}$ diffusion from QB into QW; and thus the degraded crystalline quality induces a higher non-radiative recombination. ${ }^{16}$ As a result, increasing the p-type doping range to QW and even to 2nd QB further degrades the LED performance. Note that LED with p-type
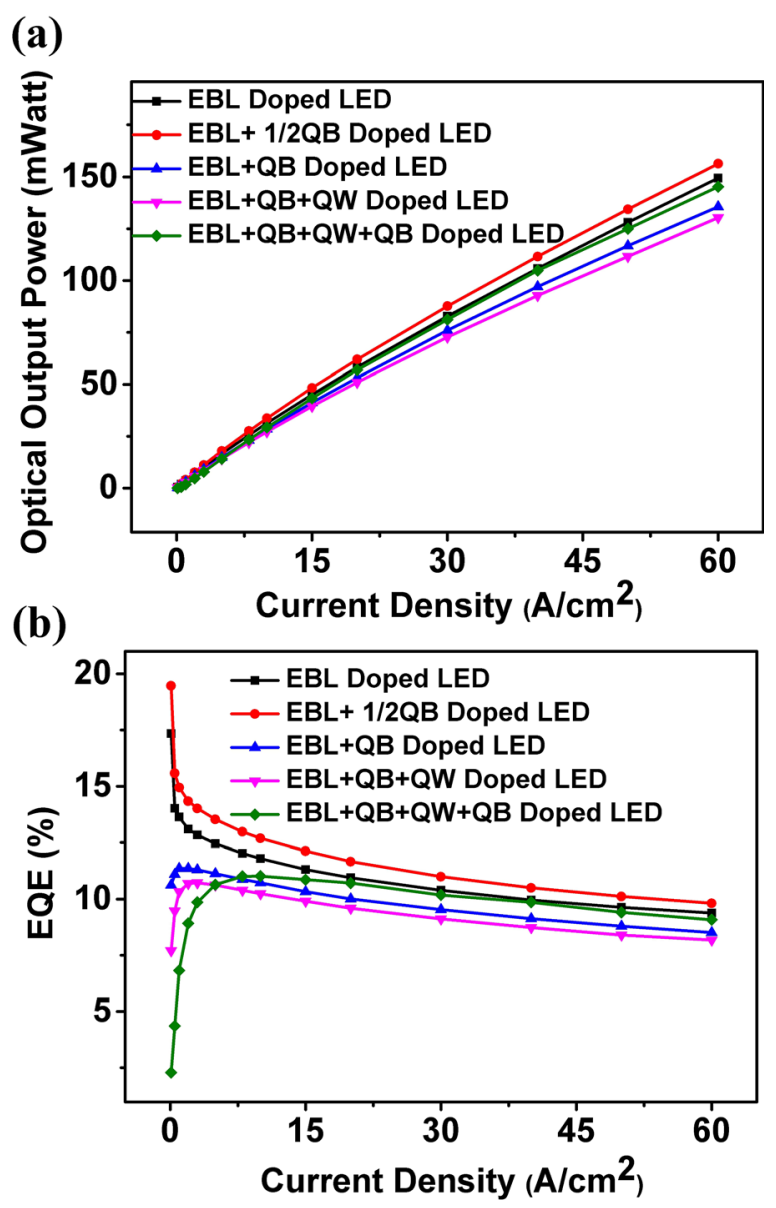

FIG. 5. (a) Optical output power versus the injection current density for LEDs with varied p-type doping ranges in depletion region, (b) experimental EQE versus the injection current density for LED with varying p-type doping ranges in the depletion region. doping at $\mathrm{EBL}+\mathrm{QB}+\mathrm{QW}+\mathrm{QB}$ has a better performance than the other two LEDs with p-type doping at EBL $+\mathrm{QB}$ and $\mathrm{EBL}+\mathrm{QB}+\mathrm{QW}$. These physical interpretations will be explained in the following analysis.

In order to support the explanation that the QW quality is degraded and more significant nonradiative recombination is caused during the p-type doping for LEDs. The corresponding experimental external quantum efficiency (EQE) is measured and presented in Fig. 5(b). From this figure, we can see that the EBL LED and EBL $+1 / 2$ QB LED have a higher EQE value, however, the EQE value drops fast as the current density increases. On the other hand, the rest of LEDs with more p-type doping region are observed to obtain an alleviated efficiency droop even though the EQE values are not high. It is worth noting that the peak-efficiency current density $\left(J_{\text {peak }}\right.$ : the current density at which EQE reaches the maximum) is $0.1,0.1,0.3,0.5$, and $0.8 \mathrm{~A} / \mathrm{cm}^{2}$ for LEDs with p-type doping at $\mathrm{EBL}, \mathrm{EBL}+1 / 2 \mathrm{QB}, \mathrm{EBL}+\mathrm{QB}$, $\mathrm{EBL}+\mathrm{QB}+\mathrm{QW}$, and $\mathrm{EBL}+\mathrm{QB}+\mathrm{QW}+\mathrm{QB}$, respectively. This value of peak-efficiency current density is a signature of the QW quality and the nonradiative recombination in QWs, such that, a larger $J_{\text {peak }}$ denotes a worse QW quality and a higher nonradiative recombination. ${ }^{17,18}$ Therefore, considering the undoped part of the last QB and the Mg memory effect that gives rise to the time delay during the $\mathrm{Mg}$ doping process, ${ }^{19}$ the $\mathrm{Mg}$ diffusion into the QW can be suppressed, and thus the LED with the p-type doping at EBL $+1 / 2 \mathrm{QB}$ shares almost the same QW quality as that with p-type doping at EBL. However, the QW quality gets worse for those LEDs with $\mathrm{Mg}$-doping at $\mathrm{EBL}+\mathrm{QB}, \mathrm{EBL}+\mathrm{QB}+\mathrm{QW}$, and $\mathrm{EBL}+\mathrm{QB}+\mathrm{QW}+\mathrm{QB}$. Consequently, the degradation of QW quality is the major cause for lowering the LED performance. Note, although the worst crystalline quality is expected for the quantum wells in the $\mathrm{EBL}+\mathrm{QB}+\mathrm{QW}+\mathrm{QB}$ doped LED, it shows a better EQE than the EBL + QB doped LED and the $\mathrm{EBL}+\mathrm{QB}+\mathrm{QW}+\mathrm{QB}$ doped LED, and this might be due to the better electron blocking effect. ${ }^{12}$

To further support this explanation, the carrier lifetime measurement for these InGaN/GaN LED devices with various p-type doping ranges in the depletion region was carried out, and the results are presented in Fig. S1 in the supplementary material. The decay time turns out to be shorter as the p-type doping range in the depletion region increases. Consequently, the reduced carrier lifetime of LED devices with increasing p-type doping range in depletion region can be a solid support to the claim that a more serious defectrelated Shockley-Read-Hall (SRH) recombination is induced when p-type doping is close to active region. To further verify the above claim, numerical simulations were performed by setting a decreased defect-related carrier lifetime for the studied devices. The calculated results are presented in Fig. S2 in the supplementary material, which exhibit the same trend as the experimental results given in Fig. 5(b). The agreement between the simulated and measured results supports that the increased nonradiative recombination appears with the increased p-type doping range close to the active region. Therefore, although the p-type doping is an effective way to enhance the hole injection and transport, the device performance enhancement is limited by the deterioration of crystal quality as the p-type doping is close to the 
active region. Thus, in order to release the full potential of $\mathrm{p}$ type doping especially in depletion region, for improving carrier transportation, $\mathrm{Mg}$ diffusion and migration have to be well controlled to guarantee the QW quality.

In conclusion, we have explored and investigated the p-type doping effect by using a model of depletion-region Mg-doping to reveal the underlying mechanism of p-type doping improvement. It was numerically found that the depletion region lies in the last $\mathrm{QB}$ and $\mathrm{EBL}$ for common EBL undoped InGaN/GaN LEDs, and it will move forward and become larger when the depletion region is designed to be p-type doping. After p-type doping in depletion region, the barrier for electrons is increased while it is reduced for holes, which contributes to the improved hole injection and transportation. Furthermore, the holes in depletion region will move under the force of the built-in electric field and strain-induced electric field. Holes obtaining sufficient energy can overcome the EBL and be stored in the p-GaN layer, while other holes will recombine nonradiatively when the equilibrium state is reached. Theoretically, it is better to have more p-type doped layers. However, experimentally we observed that if the p-type doping in depletion region is close to the active region, the quantum efficiency is reduced, which was attributed to the degrading crystal quality and the high nonradiative recombination resulting from $\mathrm{Mg}$ migration and diffusion. Therefore, p-type doping ranges near depletion region will lead to significant increase of nonradiative recombination. This was confirmed by the carrier lifetime measurements, and a good agreement has been achieved between the numerical simulation and experimental results when the p-type doping dependence of the defect-related carrier lifetime is considered in the modified numerical simulations. In summary, this work provides a detailed guidance of p-type doping in the LEDs, especially in the depletion region, to enhance carrier transportation and improve device efficiency.

See supplementary material for the parameter settings in APSYS simulations, carrier lifetime measurements, and computed EQE with revised defect concentration.
We gratefully acknowledge that this work is supported by the Singapore National Research Foundation under Grant No. NRF-CRP-6-2010-2, the Singapore Agency for Science, Technology and Research (A*STAR) SERC Pharos Program under Grant No. 15273 00025, and the National Natural Science Foundation of China (No. 61674074).

${ }^{1}$ H.-Y. Ryu and J.-I. Shim, Proc. SPIE 7939, 79390N (2011).

${ }^{2}$ Z. G. Ju, W. Liu, Z.-H. Zhang, S. T. Tan, Y. Ji, Z. B. Kyaw, X. L. Zhang, S. P. Lu, Y. P. Zhang, B. B. Zhu, N. Hasanov, X. W. Sun, and H. V. Demir, Appl. Phys. Lett. 102, 243504 (2013).

${ }^{3}$ C. H. Wang, C. C. Ke, C. Y. Lee, S. P. Chang, W. T. Chang, J. C. Li, Z. Y. Li, H. C. Yang, H. C. Kuo, T. C. Lu, and S. C. Wang, Appl. Phys. Lett. 97, 261103 (2010)

${ }^{4}$ B.-C. Lin, K.-J. Chen, C.-H. Wang, C.-H. Chiu, Y.-P. Lan, C.-C. Lin, P.-T. Lee, M.-H. Shih, Y.-K. Kuo, and H.-C. Kuo, Opt. Express 22, 463-469 (2014).

${ }^{5}$ Y. Yan Zhang and Y. An Yin, Appl. Phys. Lett. 99, 221103 (2011).

${ }^{6}$ T. Lu, S. Li, C. Liu, K. Zhang, Y. Xu, J. Tong, L. Wu, H. Wang, X. Yang, Y. Yin, G. Xiao, and Y. Zhou, Appl. Phys. Lett. 100, 141106 (2012).

${ }^{7}$ Z.-H. Zhang, Z. Kyaw, W. Liu, Y. Ji, L. Wang, S. T. Tan, X. W. Sun, and H. V. Demir, Appl. Phys. Lett. 106, 063501 (2015).

${ }^{8}$ J. Piprek, Proc. SPIE 8262, 82620E (2012).

${ }^{9}$ J. Piprek, Opt. Quantum Electron. 44, 67-73 (2012).

${ }^{10}$ L. Zhang, K. Ding, N. X. Liu, T. B. Wei, X. L. Ji, P. Ma, J. C. Yan, J. X. Wang, Y. P. Zeng, and J. M. Li, Appl. Phys. Lett. 98, 101110 (2011).

${ }^{11}$ Y. Ji, Z.-H. Zhang, S. T. Tan, Z. G. Ju, Z. Kyaw, N. Hasanov, W. Liu, X. W. Sun, and H. V. Demir, Opt. Lett. 38, 202-204 (2013).

${ }^{12}$ J. Xie, X. Ni, Q. Fan, R. Shimada, U. Ozgur, and H. Morkoc, Appl. Phys. Lett. 93, 121107 (2008).

${ }^{13}$ Y. Ji, Z.-H. Zhang, Z. Kyaw, S. Tiam Tan, Z. Gang Ju, X. Liang Zhang, W. Liu, X. Wei Sun, and H. Volkan Demir, Appl. Phys. Lett. 103, 053512 (2013).

${ }^{14}$ Y. K. Kuo, M. C. Tsai, S. H. Yen, T. C. Hsu, and Y. J. Shen, IEEE J. Quantum Electron. 46, 1214-1220 (2010).

${ }^{15}$ S.-H. Han, C.-Y. Cho, S.-J. Lee, T.-Y. Park, T.-H. Kim, S. H. Park, S. Won Kang, J. Won Kim, Y. C. Kim, and S.-J. Park, Appl. Phys. Lett. 96, 051113 (2010).

${ }^{16}$ K. Köhler, T. Stephan, A. Perona, J. Wiegert, M. Maier, M. Kunzer, and J. Wagner, J. Appl. Phys. 97, 104914 (2005).

${ }^{17}$ Light-Emitting Diodes, edited by E. F. Schubert (Cambridge University Press, 2006).

${ }^{18}$ Y. P. Zhang, Z.-H. Zhang, W. Liu, S. T. Tan, Z. G. Ju, X. L. Zhang, Y. Ji, L. C. Wang, Z. Kyaw, N. Hasanov, B. B. Zhu, S. P. Lu, X. W. Sun, and H. V. Demir, Opt. Express 23, A34-A42 (2015).

${ }^{19}$ Y. Ohba and A. Hatano, J. Cryst. Growth 145, 214-218 (1994). 